\title{
Effect of influenza vaccine on COVID-19 mortality: a retrospective study
}

\author{
Marcello Candelli ${ }^{1}$ (1) Giulia Pignataro ${ }^{1} \cdot$ Enrico Torelli ${ }^{1} \cdot$ Antonio Gulli $^{2} \cdot$ Enrico Celestino Nista $^{1} \cdot$ Martina Petrucci $^{1}$. \\ Angela Saviano ${ }^{1}$ - Debora Marchesini ${ }^{1}$ - Marcello Covino ${ }^{1}$. Veronica Ojetti ${ }^{1} \cdot$ Massimo Antonelli ${ }^{2}$. \\ Antonio Gasbarrini ${ }^{3} \cdot$ Francesco Franceschi $^{1}$
}

Received: 27 November 2020 / Accepted: 8 March 2021 / Published online: 20 March 2021

(c) Società Italiana di Medicina Interna (SIMI) 2021

\begin{abstract}
It has been proposed that vaccines may exert an unspecific protective effect against infectious agents, different than expected. Coronavirus disease 2019 (COVID-19) is a pandemic infection with high mortality in older patients due to severe acute respiratory syndrome coronavirus 2 (SARS-CoV-2). The high number of vaccinations may be one of the reasons why children show a lower susceptibility to SARS-CoV-2 infection and milder severity when compared to adults. We have designed a study aimed at investigating whether the influenza vaccine may reduce the susceptibility and severity of SARS-CoV-2 infection. We retrospectively enrolled 635 patients who accessed our Emergency Department from March 1st to June 30th, 2020, and were diagnosed with COVID-19 infection confirmed by an RT-PCR on an oropharyngeal swab. Clinical data, outcomes, and influenza vaccination status were collected from the electronic medical records of our Hospital. We also used data from the Italian Health Ministry to compare the prevalence of flu vaccination among the general population of the Lazio Region and our enrolled patients. We then compared clinical outcomes between vaccinated and non-vaccinated patients, by univariate and multivariate analysis. COVID-19-positive patients older than 65 years reported a lower prevalence of flu vaccination when compared to the general population residing in the Lazio $(p=0.004)$. After correction for gender, age, and comorbidities, we found a lower risk of death at 60 days in patients with flu vaccination than in not vaccinated patients $(p=0.001)$. Our study shows that flu vaccination could reduce the mortality of COVID-19. Prospective studies are needed to confirm this result.
\end{abstract}

Keywords Influenza vaccine $\cdot$ COVID- $19 \cdot$ Mortality $\cdot$ Susceptibility $\cdot$ Comorbidities

\section{Introduction}

Severe acute respiratory syndrome coronavirus 2 (SARS$\mathrm{CoV}-2)$ is a recently isolated virus that causes the coronavirus disease 2019 (COVID-19), a pathology with

Marcello Candelli

marcello.candelli@policlinicogemelli.it

1 Emergency Medicine Department, Fondazione Universitaria Policlinico Gemelli-IRCCS-Università Cattolica del Sacro Cuore, Largo Agostino Gemelli 8, 00135 Rome, Italy

2 Anestesiology and Resuscitation Department, Fondazione Universitaria Policlinico Gemelli-IRCCS-Università Cattolica del Sacro Cuore, Largo Agostino Gemelli 8, 00135 Rome, Italy

3 Internal Medicine Department, Fondazione Universitaria Policlinico Gemelli-IRCCS-Università Cattolica del Sacro Cuore, Largo Agostino Gemelli 8, 00135 Rome, Italy extremely variable clinical expression, going from asymptomaticity to severe respiratory failure [1]. Older age is an independent risk factor for the susceptibility and mortality of COVID-19 even after correction for comorbidity and gender [1]. Interestingly, children generally show particularly mild clinical manifestations and very low mortality [2]. Among all possible factors able to explain a lower severity of COVID-19 in children, there is a non-specific response of the immune system due to previous vaccinations to other pathogens [3], especially respiratory viruses [4]. The theory is that the administration of a vaccine could lead to an increased or reduced risk to acquire infections caused by other pathogens $[5,6]$. The hypothesized pathophysiological mechanisms underlying the interaction between influenza vaccine and coronavirus infection are structural similarities in ligands for cell receptors, antibody cross-reactivity, and a non-specific cell-mediated response [4, 7-9]. Several authors have studied the effect 
of influenza (flu) and pneumococcal vaccination on the susceptibility and mortality of COVID-19, with conflicting results $[10,11]$.

This is why we have designed a study aimed at verifying whether influenza vaccination may influence the clinical course of COVID-19.

\section{Methods}

We retrospectively enrolled 635 consecutive patients who resulted positive to SARS-CoV-2 in our emergency department (ED), Fondazione Policlinico Gemelli-IRCCS of Rome, from March 1st, 2020 to June 30th, 2020. COVID-19 diagnosis was confirmed by a real-time reverse transcriptase polymerase-chain-reaction (RT-PCR) on nasopharyngeal and oropharyngeal swabs.

Five patients were excluded by the study because they denied their consensus on data treatment, while 28 patients were excluded because it was not possible to collect data on influenza vaccination status. For all other 602 participants, we collected demographic data. Clinical information, epidemiological data, comorbidities, radiological images, laboratory findings, and clinical outcomes (mortality at 60 days, length of hospitalization, days spent in Intensive Care Unit (ICU) and need for admission in ICU, need for endotracheal intubation (ETI) were also collected from the electronic medical records of our hospital. We then used data on vaccination status to create two groups, such as previously vaccinated and unvaccinated patients and performed a statistical analysis to investigate all factors associated with different outcomes.

This study was approved by the Ethical Committee of the Catholic University of Rome (protocol number 3327) and has been conducted following the declaration of Helsinki.

\section{Statistical analysis}

Statistical analysis was performed using IBM SPSS 20 software. Data were expressed as mean \pm standard deviation. Prevalence was expressed as a percentage. The comparison between groups was performed by Mann-Whitney $U$ test for non-parametric data; a comparison between categorical variables was performed using the Chi-square test or the Fisher's exact test as appropriate. A multivariate analysis with logistic regression was performed to correct for the main confounding factors. We performed two models with death at 60 days and ETI as the outcomes and flu vaccination status as the independent variable together with other demographic and clinical covariates (gender, age, and comorbidities). Values of $p<0.05$ were considered statistically significant.

\section{Results}

We finally enrolled 602 patients (390 males and 215 females, mean age: $60.6 \pm 16.3$ years) with a diagnosis of COVID-19 infection confirmed by RT-PCR on an oropharyngeal and nasopharyngeal swab. In Table 1 were described the main laboratory values at admission in the ED. Two hundred and sixty-two patients did not show any investigated chronic comorbidity. On the other hand, 340 patients presented one or more comorbidities (Table 2). Among patients affected by hypertension or chronic heart disease, 194 were using inhibitors of angiotensin-converting enzyme (ACEi) or angiotensin II receptor blockers (ARBs).

Ninth-two (15.3\%) patients were admitted for at least $24 \mathrm{~h}$ in an Intensive Care Unit (ICU) during hospitalization. After a 60-day follow-up period, 44 (7.3\%) patients were deceased. The average length of hospitalization was $19.0 \pm 15$ days. The average length of stay in ICU was $12.0 \pm 11$ days.

The most common referred symptoms during the disease were fever $(570 / 602,94.7 \%)$, dry cough $(450 / 602$, $74.7 \%)$, dyspnea $(390 / 602,64.8 \%)$, and weakness $(340 / 602,56.5 \%)$, while the mean value of $\mathrm{PaO} 2 / \mathrm{FiO} 2$ in the emergency room was $339 \pm 115$.

Pneumonia was diagnosed by chest X-ray in 419 , (69.6\%) patients. In 302 (50.2\%) patients, we observed the involvement of both lungs. In Table 3 is described the need for oxygen supplementation and ventilation.

\section{Vaccinated versus unvaccinated COVID-19 patients}

The prevalence of influenza vaccination was $24.9 \%$ (150/602) in COVID-19 patients. No difference in gender was observed between vaccinated and not vaccinated patients: male patients were $88 / 150(58.7 \%)$ vs $302 / 452$ $(66.8 \%)$, respectively $(p=0.07)$. The mean age of vaccinated patients resulted significantly higher when compared to unvaccinated patients $(70.4 \pm 16$ vs $57.3 \pm 15$ years; $p<0.0001)$. Vaccinated patients showed a higher prevalence of at least one chronic disease $(110 / 150,73.3 \%)$ than controls $(230 / 452,50.9 \%$; $p<0.0001$; OR 2.65; IC 95\% $1.768-3.984)$. In particular, we found a higher prevalence in vaccinated people of hypertension, type 2 diabetes, chronic heart disease, chronic congestive cardiac failure, COPD, and neoplasms ( 13.3 vs $6.2 \%, p=0.005$; Table 2 ). Twenty-eight $(18.7 \%)$ vaccinated patients need of admission in ICU versus $64(14.2 \%)$ of unvaccinated COVID-19 patients $(p=0.18)$. No difference in the number of patients deceased at 60 days from diagnosis $(8 / 150,5.3 \%$ vs $36 / 416,8 \% ; p=0.28)$ was found. We found that vaccinated patients had a significantly 
Table 1 COVID-19 patient's laboratory characteristics and comparison between vaccinated and not vaccinated COVID-19 patients

\begin{tabular}{|c|c|c|c|c|c|}
\hline & Enrolled patients & Normal values & Vaccinated patients & Unvaccinated patients & $p$ \\
\hline BUN (mg/dL) & $19 \pm 15$ & $10-23$ & $21.9 \pm 16$ & $18.5 \pm 15$ & $<0.0001$ \\
\hline Creatinin (mg/dL) & $1.5 \pm 5.9$ & $0.67-1.17$ & $1.1 \pm 1$ & $1.2 \pm 1$ & 0.266 \\
\hline Sodium $(\mathrm{mmol} / \mathrm{L})$ & $138 \pm 8$ & $135-145$ & $138 \pm 4$ & $138 \pm 9$ & 0.203 \\
\hline Potassium (mmol/L) & $4.0 \pm 0.5$ & $3.5-5$ & $3.9 \pm 0.5$ & $4.0 \pm 0.5$ & 0.572 \\
\hline CPK (UI/L) & $35 \pm 34$ & $7-45$ & $151 \pm 142$ & $187 \pm 333$ & 0.567 \\
\hline $\mathrm{LDH}(\mathrm{UI} / \mathrm{L})$ & $178 \pm 298$ & $30-170$ & $339 \pm 198$ & $309 \pm 123$ & 0.525 \\
\hline $\mathrm{CRP}(\mathrm{mg} / \mathrm{L})$ & $316 \pm 146$ & $<250$ & $86 \pm 87$ & $66 \pm 67$ & 0.882 \\
\hline Procalcitonin (ng/mL) & $71 \pm 73$ & $<5$ & $0.3 \pm 1$ & $2 \pm 19$ & 0.306 \\
\hline $\mathrm{Hb}(\mathrm{g} / \mathrm{dL})$ & $1.6 \pm 16$ & $<0.5$ & $13.6 \pm 2$ & $14.3 \pm 2$ & 0.823 \\
\hline $\operatorname{PLT}\left(\times 10^{\wedge} 9 / \mathrm{L}\right)$ & $14 \pm 1$ & $13-17$ & $217 \pm 85$ & $208 \pm 81$ & 0.252 \\
\hline Leukocytes $\left(\times 10^{\wedge} 9 / \mathrm{L}\right)$ & $210 \pm 82$ & $150-450$ & $7.260 \pm 4.330$ & $8.501 \pm 2.847$ & 0.159 \\
\hline Neutrophils $\left(\times 10^{\wedge} 9 / \mathrm{L}\right)$ & $8.190 \pm 2475$ & $4.000-10.000$ & $5.291 \pm 3.642$ & $4.893 \pm 2.766$ & 0.298 \\
\hline Neutrophils \% & $4993 \pm 3011$ & $2.000-7.000$ & $72.5 \pm 14$ & $71.5 \pm 13$ & 0.229 \\
\hline Lymphocytes $\left(\times 10^{\wedge} 9 / \mathrm{L}\right)$ & $72 \pm 13$ & $40-80$ & $1.447 \pm 2.331$ & $1.228 \pm 0.790$ & 0.549 \\
\hline Lymphocytes \% & $1.282 \pm 1.390$ & $1.000-3.000$ & $20.3 \pm 13$ & $20.1 \pm 10$ & 0.320 \\
\hline D-Dimer (ng/mL) & $20 \pm 11$ & $20-40$ & $2016 \pm 3484$ & $2137 \pm 5046$ & 0.226 \\
\hline Fibrinogen (mg/dL) & $2106 \pm 4690$ & $<500$ & $515 \pm 188$ & $497 \pm 170$ & 0.331 \\
\hline Troponin (ng/dL) & $501 \pm 174$ & $200-400$ & $18 \pm 23$ & $28 \pm 205$ & 0.015 \\
\hline NTproBNP (pg/mL) & $26 \pm 177$ & $<57$ & $1449 \pm 3600$ & $393 \pm 771$ & $<0.0001$ \\
\hline
\end{tabular}

$B U N$ blood urea nitrogen, $A L T$ alanine transferase, $C P K$ creatine phosphokinase, $L D H$ lactic dehydrogenase, $C R P$ c-reactive protein $H b$ hemoglobin, $P L T$ platelets, NTproBNP N-terminal pro brain natriuretic peptide

Table 2 Comparison of comorbidities prevalence in vaccinated and not vaccinated patients with COVID-19

\begin{tabular}{lllcc}
\hline Comorbidity $N(\%)$ & Enrolled patients & Vaccinated patients & Unvaccinated patient & $P$ \\
\hline Hypertension & $248(41.2)$ & $96(64.0)$ & $152(33.6)$ & $<0.0001$ \\
Obesity & 80 & $18(12.0)$ & $62(13.7)$ & 0.6 \\
Diabetes & $66(11)$ & $26(17.3)$ & $40(8.8)$ & 0.004 \\
CHD & 50 & $24(16.0)$ & $26(5.7)$ & $<0.0001$ \\
CND & 48 & $9(6.0)$ & $39(8.3)$ & 0.35 \\
Neoplasms & 48 & $20(13.3)$ & $28(6.2)$ & 0.005 \\
COPD & 46 & $22(14.7)$ & $24(5.3)$ & 0.0002 \\
CCF & $20(13.3)$ & $16(3.5)$ & $<0.0001$ \\
AF & 36 & $10(6.7)$ & $18(4.0)$ & 0.18 \\
OPD & 28 & $4(2.7)$ & $10(2.2)$ & 0.75 \\
Renal failure & 14 & $2(1.3)$ & $11(2.4)$ & 0.53 \\
\hline
\end{tabular}

Chronic heart disease (CHD), Chronic congestive heart failure (CCF), Chronic obstructive pulmonary disease (COPD), Atrial fibrillation (AF), Other chronic pulmonary disease (OPD), Chronic neurological disease (CND) higher length of hospitalization than patients not vaccinated $(21.4 \pm 16$ vs $18.2 \pm 14$ days; $p=0.04)$. No difference between vaccinated and non-vaccinated patients was found in days spent in ICU $(11.9 \pm 10$ vs $12.7 \pm 12$ days; $p=0.73)$. Table 1 describes the results of the comparison of laboratory data between the groups. No difference was found between the groups in $\mathrm{PaO} 2 / \mathrm{FiO} 2$ ratio: $332 \pm 108$ in vaccinated patients vs $342 \pm 116$ in not vaccinated patients $(p=0.325)$. Moreover, the prevalence of a $\mathrm{PaO} 2 / \mathrm{FiO} 2$ ratio less than
300 was similar between vaccinated vs not vaccinated subjects (33 vs 29\%; $p=0.34$ ). No difference in the incidence of pneumonia at chest X-ray was found in vaccinated patients when compared to patients in the other group (113/150, $75.3 \%$ vs $306 / 452,67.7 \% ; p=0.08)$. Table 3 shows the number of patients needing oxygen or ventilation between the studied groups. No difference in the number of patients who need high oxygen flux, non-invasive ventilation (NIV), or ETI was found. 
Table 3 Maximal need for oxygen supplementation, not invasive, and invasive ventilation in vaccinated and not vaccinated COVID-19 patients

\begin{tabular}{llll}
\hline $\begin{array}{l}\text { Maximal need for } \\
\text { oxygen }\end{array}$ & Vaccinated $N(\%)$ & Unvaccinated $N(\%)$ & $p$ \\
\hline No oxygen & $53(35)$ & $193(43)$ & 0.11 \\
$\begin{array}{l}\text { Nasal high flux } \\
\text { oxygen or venture }\end{array}$ & $73(49)$ & $183(40)$ & 0.08 \\
$\quad$ & & & \\
mask & $12(8)$ & $38(8.4)$ & 0.89 \\
ETV & $12(8)$ & $38(8.4)$ & 0.89 \\
\hline
\end{tabular}

NIV non-invasive ventilation (Helmet or facial mask), ETI endotracheal intubation

To investigate whether the high difference between groups in age, gender, and comorbidity could occult an association between the previous flu vaccine and prognosis in COVID-19 patients, we perform a multinomial logistic regression analysis in which we evaluated death at 60 days from diagnosis and the need for ETI as outcomes and vaccination status, gender, age, and comorbidities found significantly different between groups at univariate analysis as independent variables and covariates. After correction for gender, age, and comorbidities (chronic ischemic heart disease, chronic congestive heart failure, chronic obstructive pulmonary disease, neoplasms), we found that flu vaccination was independently associated with a lower risk of death at 60 days ( $p<0.001$; OR 0.2; IC 95\% 0.082-0.510), but not to the need for ETI in COVID-19 patients (Table 4).

\section{Discussion}

Several studies investigating the correlation among influenza vaccination, susceptibility, and outcomes of COVID-19 were recently published with discordant results. Even if the susceptibility to SARS-CoV-2 was not the aim of our study, we can use data from the Italian Health Ministry and from the Italian National Institute of Statistic (Istat) to compare the prevalence of flu vaccination among the general population and patients enrolled in our study [12-14].

When we compared data between the population residing in Lazio and COVID-19 patients, we found a significant difference among the vaccinated and unvaccinated subjects. In younger people (18-65 years old), flu vaccination seems to increase susceptibility to COVID-19 infection; on the contrary, when analyzing data from older people ( $>65$ years old), we found a protective effect of vaccination on acquiring COVID-19 infection (Table 5). However, these data are not corrected for gender and comorbidities. Moreover, in Italy, flu vaccination is free of charge for people with diabetes, cardiovascular, pulmonary, and other chronic diseases, even in those less than 64 years old. These patients could be at higher risk of infection because they spend more time in the hospital than healthy people and may have an immune system lesser responsive to infections and with a lower ability to clear off the viral particles from the respiratory tree. Finally, they were at higher risk of complications, and a higher number of young not vaccinated subjects could be asymptomatic or mildly symptomatic and did not access our ED. Our study population is extremely selected (we enrolled only patients

Table 4 Need for ETI in vaccinated vs unvaccinated patients at multivariate analysis with correction for gender, age, and comorbidities

\begin{tabular}{llllrr}
\hline & Need for ETI (50) & No need for ETI (552) & $p$ & OR & 95\% IC limits \\
\hline Vaccination $N(\%)$ & $12(24)$ & $138(25)$ & 0.42 & 0.73 & $0.346-1.559$ \\
\hline & Patients dead at 60 days (44) & Patients alive at 60 days (558) & $p$ & OR & $95 \%$ IC limits \\
\hline Vaccination $N(\%)$ & $8(18.2)$ & $142(25.4)$ & 0.001 & 0.20 & $0.082-0.510$ \\
\hline
\end{tabular}

ETI endotracheal intubation. Comorbidities evaluated for analysis: congestive chronic heart failure, Chronic obstructive pulmonary disease, Cardiac heart disease, Diabetes, hypertension, and neoplasms

Table 5 Prevalence of 20192020 flu vaccination in patients with confirmed COVID-19 and the general population of the Lazio by age

\begin{tabular}{lllll}
\hline Age (years) & $>18$ & $18-64$ & $>64$ & $>0$ \\
People resident in Lazio $N$ & $4,929,397$ & $3,628,643$ & $1,291,754$ & $5,865,544$ \\
Vaccinated $N(\%)$ & $937,931(19)$ & $257,177(7)$ & $680,754(52.7)$ & $950,218(16.2)$ \\
COVID-19 patients $N$ & 602 & 358 & 244 & 602 \\
COVID-19 vaccinated $N(\%)$ & $150(24.9)$ & $44(12.3)$ & $106(43.4)$ & $150(24.9)$ \\
$p$ & 0.002 & 0.0001 & 0.004 & $<0.0001$ \\
OR & 1.40 & 1.83 & 0.69 & 1.72 \\
IC 95\% & $1.17-1.69$ & $1.34-2.51$ & $0.53-0.89$ & $1.43-2.06$ \\
\hline
\end{tabular}

OR odds ratio, IC $95 \%$ confidence interval 
who need admission to ED) and does not include patients with mild or absent symptoms. Moreover, age, gender, and other chronic diseases are risk factors for severe disease and not for infection. For all these reasons, the result of a comparison between the general population and enrolled patients cannot be used to evaluate the difference in susceptibility but shows the different distribution of vaccination status between the general population and patients affected by more severe disease.

When we analyzed data between COVID-19 vaccinated and unvaccinated patients with univariate analysis, we did not found a significant difference in gender prevalence. On the contrary, the mean age was significantly higher in vaccinated patients than in not vaccinated. This finding is not surprising, since the rate of influenza vaccination in Italy increases with age, particularly after 65 years, and the phenomenon is also reflected in our population. The higher prevalence of comorbidity in vaccinated patients was, also, not surprising, since in Italy flu vaccination is free of charge and strongly recommended by public health authorities in patients with chronic diseases, while the prevalence of comorbidities increases with age. The older age and the higher number of comorbidities (cardiac diseases, neoplasms, and chronic diseases in general) may also explain the longer length of hospitalization and the significant difference for PCR, hemoglobin, and NTproBNP blood levels observed in vaccinated patients. We did not find a difference in the prevalence of pneumonia at a chest X-ray. On the contrary, we were surprised by the absence, at univariate analysis, of increased death rate and greater need for oxygen supplementation in vaccinated patients than we expected, considering the higher mean age and the higher prevalence of severe comorbidities. Surprisingly, when we performed a multivariate logistic regression to correct for confounding factors (age, gender, and all comorbidities), we found that flu vaccination was associated with lower mortality at 60 days.

An unspecific effect of infection and vaccination on the immune system and susceptibility to other infections has been previously described, even though data are discordant. In a recent study, the influenza vaccine slightly reduces the odds to be infected by other respiratory diseases [5], while an older study found an increase of other respiratory viral infections in vaccinated children [6]. Moreover, other researchers did not found any effect of vaccination on the incidence of other respiratory illnesses [15]. As regards susceptibility to acquiring COVID-19 infection, it was described that flu vaccination was associated with a reduced risk of infection and it was estimated a $28 \%$ reduction in death rate in older people with a $10 \%$ increase in vaccination coverage $[16,17]$. Large population studies have shown conflicting results [18-21]. Jehi et al. correlated flu vaccination to an increased rate of hospitalization. However, in this study, no correction for age, comorbidities, and gender was performed and the mean age between hospitalized and not hospitalized patients was different (64 vs 51 years in the study cohort and 65 vs 46 years in validation one) [17]. Murillo-Zamora et al. did not find a correlation between the severity of disease (evaluated as hospitalization for pneumonia needing oxygen) and previous flu vaccination. These authors also did not correct data for gender and age, factors that were found to be significantly different between vaccinated and not vaccinated patients in their study [18]. Ortiz-Prado et al. did not observe a correlation between mortality and flu vaccination in COVID-19 patients but once again, data were not corrected for confounding factors and the very low number of vaccinated patients $(<1 \%$ of enrolled) did not allow to draw any significant conclusions [19]. Pobadour-Plou et al. did not find a correlation between flu vaccination and mortality after correction for age in both men and women but they did not correct data for comorbidities [20]. In a very large Brazilian study, after correction for age, gender, and comorbidities, a protective effect of flu vaccination on the severity, evaluated as needing ICU admission or respiratory support, and mortality was described. However, in this study, only $84 \%$ of enrolled patients received a molecularly confirmed diagnosis of COVID-19, and about $8 \%$ of patients were vaccinated after the onset of COVID-19 symptoms [21]. In our study, we corrected our data for comorbidity, gender, and age. All patients received the flu shot weeks before the onset of COVID-19 symptoms and all diagnoses were performed through nasopharyngeal and oropharyngeal swabs. Interestingly, in an epidemiological study performed on an Italian population, the flu vaccine was associated with reduced susceptibility to COVID-19. However, in this study, the death rate was affected by flu vaccination only in a small group of patients who received the vaccine in the period immediately preceding the onset of the outbreak [11]. In our study, we only included symptomatic patients who presented themselves to the Emergency Department. Ragni P et al., unlike us, enrolled all swab-positive patients, both symptomatic and asymptomatic of an entire Italian province. The differences in results with our study can be explained by the different enrollment criteria. Finally, another Italian epidemiological study found a negative correlation in patients older than 65 years between the flu shot and death related to COVID-19 [22]. This finding is similar to the results of our study. Despite our study is retrospective and bias cannot be completely excluded, we may speculate that the positive effect of influenza vaccination is related to protection for flu disease. Flu induces an increased expression of ACE2 receptors in pulmonary alveolar cells and may increase the severity of a subsequent SARS-CoV-2 infection [23], while preventing influenza utilizing vaccine may reduce the viral load and the severity of the COVID-19-related disease. On the other hand, the flu vaccine activates the Toll-Like Receptor 7 (TLR7) on the surface of macrophages, dendritic cells, 
and neutrophils and induces, via NFKB, a faster response to the virus in case of infection, improving both innate and adaptive immunity [24].

\section{Conclusion}

Flu vaccine seems to decrease mortality of COVID-19 at 60 days of all ages. Further prospective studies are needed to confirm our data and explain the molecular bases of those results. Meanwhile, we recommended flu vaccination in all patients especially those older than 65 years and with chronic comorbidities.

Acknowledgements GEMELLI Against COVID-19 Group: Valeria Abbate, Nicola Acampora, Giovanni Addolorato, Fabiana Agostini, Maria E. Ainora, Elena Amato, Gloria Andriollo, Brigida E. Annicchiarico, Mariangela Antonelli, Gabriele Antonucci, Armuzzi Alessandro, Christian Barillaro, Fabiana Barone, Rocco D. A. Bellantone, Andrea Bellieni, Andrea Benicchi, Francesca Benvenuto, Filippo Berloco, Roberto Bernabei, Antonio Bianchi, Luigi M. Biasucci, Stefano Bibbò, Federico Biscetti, Nicola Bonadia, Alberto Borghetti, Giulia Bosco, Silvia Bosello, Vincenzo Bove, Giulia Bramato, Vincenzo Brandi, Dario Bruno, Maria C. Bungaro, Alessandro Buonomo, Livia Burzo, Angelo Calabrese, Andrea Cambieri, Giulia Cammà, Marcello Candelli, Gennaro Capalbo, Lorenzo Capaldi, Esmeralda Capristo, Luigi Carbone, Silvia Cardone, Angelo Carfî, Annamaria Carnicelli, Cristiano Caruso, Francesco A. Casciaro, Lucio Catalano, Roberto Cauda, Andrea L. Cecchini, Lucia Cerrito, Michele Ciaburri, Rossella Cianci, Sara Cicchinelli, Arturo Ciccullo, Francesca Ciciarello, Antonella Cingolani, Maria C. Cipriani, Gaetano Coppola, Andrea Corsello, Federico Costante, Marcello Covino, Stefano D’Addio, Alessia D'Alessandro, Maria E. D'alfonso, Emanuela D'Angelo, Francesca D'Aversa, Fernando Damiano, Tommaso De Cunzo, Giuseppe De Matteis, Martina De Siena, Francesco De Vito, Valeria Del Gatto, Paola Del Giacomo, Fabio Del Zompo, Davide Della Polla, Luca Di Gialleonardo, Simona Di Giambenedetto, Roberta Di Luca, Luca Di Maurizio, Alex Dusina, Alessandra Esperide, Domenico Faliero, Cinzia Falsiroli, Massimo Fantoni, Annalaura Fedele, Daniela Feliciani, Andrea Flex, Evelina Forte, Francesco Franceschi, Laura Franza, Barbara Funaro, Mariella Fuorlo, Domenico Fusco, Maurizio Gabrielli, Eleonora Gaetani, Antonella Gallo, Giovanni Gambassi, Matteo Garcovich, Antonio Gasbarrini, Irene Gasparrini, Silvia Gelli, Antonella Giampietro, Laura Gigante, Gabriele Giuliano, Giorgia Giuliano, Bianca Giupponi, Elisa Gremese, Caterina Guidone, Amerigo Iaconelli, Angela Iaquinta, Michele Impagnatiello, Riccardo Inchingolo, Raffaele Iorio, Immacolata M. Izzi, Cristina Kadhim, Daniele I. La Milia, Francesco Landi, Giovanni Landi, Rosario Landi, Massimo Leo, Antonio Liguori, Rosa Liperoti, Marco M. Lizzio, Maria R. Lo Monaco, Pietro Locantore, Francesco Lombardi, Loris Lopetuso, Valentina Loria, Angela R. Losito, Andrea Lupascu, Noemi Macerola, Giuseppe Maiuro, Francesco Mancarella, Francesca Mangiola, Alberto Manno, Debora Marchesini, Giuseppe Marrone, Ilaria Martis, Anna M. Martone, Emanuele Marzetti, Maria V. Matteo, Luca Miele, Alessio Migneco, Irene Mignini, Alessandro Milani, Domenico Milardi, Massimo Montalto, Flavia Monti, Davide Moschese, Barbara P. L. Mothaenje, Celeste A. Murace, Rita Murri, Marco Napoli, Elisabetta Nardella, Gerlando Natalello, Simone M. Navarra, Antonio Nesci, Alberto Nicoletti, Tommaso Nicoletti, Rebecca Nicolò, Nicola Nicolotti, Enrico C. Nista, Eugenia Nuzzo, Veronica Ojetti, Francesco C. Pagano, Cristina Pais, Alfredo Papa, Luigi G. Papparella, Mattia Paratore, Giovanni Pecorini, Simone Perniola, Erika Pero, Giuseppe Perrinello, Luca
Petricca, Martina Petrucci, Chiara Picarelli, Andrea Piccioni, Giulia Pignataro, Raffaele Pignataro, Marco Pizzoferrato, Fabrizio Pizzolante, Roberto Pola, Caterina Policola, Maurizio Pompili, Valerio Pontecorvi, Francesca Ponziani, Valentina Popolla, Enrica Porceddu, Angelo Porfidia, Giuseppe Privitera, Gabriele Pulcini, Simona Racco, Francesca Raffaelli, Gian L. Rapaccini, Luca Richeldi, Emanuele Rinninella, Sara Rocchi, Stefano Romano, Federico Rosa, Laura Rossi, Raimondo Rossi, Enrica Rossini, Elisabetta Rota, Fabiana Rovedi, Gabriele Rumi, Andrea Russo, Luca Sabia, Andrea, Salerno, Sara Salini, Lucia Salvatore, Dehara Samori, Maurizio Sanguinetti, Luca Santarelli, Paolo Santini, Angelo Santoliquido, Francesco Santopaolo, Michele C. Santoro, Francesco Sardeo, Caterina Sarnari, Luisa Saviano, Tommaso Schepis, Francesca Schiavello, Giancarlo Scoppettuolo, Luisa Sestito, Carlo Settanni, Valentina Siciliano, Benedetta Simeoni, Andrea Smargiassi, Domenico Staiti, Leonardo Stella, Eleonora Taddei, Rossella Talerico, Enrica Tamburrini, Claudia Tarli, Pietro Tilli, Enrico Torelli, Matteo Tosato, Alberto Tosoni, Luca Tricoli, Marcello Tritto, Mario Tumbarello, Anita M. Tummolo, Federico Valletta, Giulio Ventura, Lucrezia Verardi, Lorenzo Vetrone, Giuseppe Vetrugno, Elena Visconti, Raffaella Zaccaria, Lorenzo Zelano, Lorenzo Zileri Dal Verme, Giuseppe Zuccalà.

Funding No external funding was used to perform the study.

\section{Declarations}

Conflict of interest The authors declare that they have no competing interests.

Ethical approval This study was approved by the Ethical Committee of the Catholic University of Rome (protocol number 3327) and has been conducted in accordance with the Declaration of Helsinki.

Statement of human and animal rights The protocol was approved by the Hospital Institutional Review Board.

Consent to participate An informed consent was signed by each patient for the treatment of clinical data for research purposes.

\section{References}

1. Zhou F, Yu T, Du R et al (2020) Clinical course and risk factors for mortality of adult inpatients with COVID-19 in Wuhan, China: a retrospective cohort study. Lancet 395:1054-1062. https://doi. org/10.1016/S0140-6736(20)30566-3 (Epub 2020 Mar 11)

2. Götzinger F, Santiago-García B, Noguera-Julián A et al (2020) COVID-19 in children and adolescents in Europe: a multinational, multicentre cohort study. Lancet Child Adolesc Health 4:653-661. https://doi.org/10.1016/S2352-4642(20)30177-2 (Epub 2020 Jun 25)

3. Yu Y (2020) Herd immunization with childhood vaccination may provide protection against COVID-19. Acta Microbiol Immunol Hung. https://doi.org/10.1556/030.2020.01207. Epub ahead of print.

4. Wolff GG (2020) Influenza vaccination and respiratory virus interference among Department of Defense personnel during the 2017-2018 influenza season. Vaccine 38:350-354. https://doi.org/ 10.1016/j.vaccine.2019.10.005 (Epub 2019 Oct 10)

5. Cowling BJ, Fang VJ, Nishiura $\mathrm{H}$ et al (2012) Increased risk of noninfluenza respiratory virus infections associated with receipt of inactivated influenza vaccine. Clin Infect Dis 54:1778-1783. https://doi.org/10.1093/cid/cis307 (Epub 2012 Mar 15) 
6. Feng S, Fowlkes AL, Steffens A, Finelli L, Cowling BJ (2017) Assessment of virus interference in a test-negative study of influenza vaccine effectiveness. Epidemiology 28:514-524. https://doi. org/10.1097/EDE.0000000000000670

7. Koutsakos M, Wheatley AK, Loh L et al (2018) Circulating TFH cells, serological memory, and tissue compartmentalization shape human influenza-specific B cell immunity. Sci Transl Med 10:8405. https://doi.org/10.1126/scitranslmed.aan8405

8. Zeng Q, Langereis MA, van Vliet ALW, Huizinga EG, de Groot RJ (2008) Structure of coronavirus hemagglutinin-esterase offers insight into corona and influenza virus evolution. Proc Natl Acad Sci 105:9065-69. https://doi.org/10.1073/pnas.0800502105

9. Matrosovich M, Herrler G, Klenk HD et al (2015) Sialic acid receptors of viruses. Top Curr Cem 367:1-28. https://doi.org/10. 1007/128_2013_466

10. Patwardhan A, Ohler A (2021) The flu vaccination may have a protective effect on the course of COVID-19 in the pediatric population: when does severe acute respiratory syndrome coronavirus 2 (SARS-CoV-2) meet influenza? Cureus 13:e12533. https://doi. org/10.7759/cureus. 12533

11. Ragni P, Marino M, Formisano D et al (2020) Association between exposure to influenza vaccination and COVID-19 diagnosis and outcomes. Vaccines (Basel) 8:675. https://doi.org/10. 3390/vaccines 8040675

12. Website of Italian National Institute of Statistic (Istat) (2020) http://dati.istat.it/Index.aspx ?lang=en\&SubSessionId $=$ cb32d 7a0-c588-4872-99f4-6730619bf1c8 Accessed 24 Nov 2020.

13. Website of Italian ministry of Health (2020) http://www.salute. gov.it/portale/documentazione/p6_2_8_3_1.jsp?lingua=italiana\& $\mathrm{id}=19$. Accessed 24 Nov 2020

14. Website of Italian Ministry of Health (2020) http://www.salute. gov.it/imgs/C_17_tavole_19_5_0_file.pdf. Accessed 24 Nov 2020

15. Jehi L, Ji X, Milinovich A, Erzurum S et al (2020) Individualizing risk prediction for positive coronavirus disease 2019 testing: results from 11,672 patients. Chest 158:1364-1375. https://doi. org/10.1016/j.chest.2020.05.580

16. Zanettini C, Omar M, Dinalankara W et al (2020) Influenza vaccination and COVID-19 mortality in the USA. MedRxiv. https:// doi.org/10.1101/2020.06.24.20129817.06.24.20129817

17. Jehi L, Ji X, Milinovich A, Erzurum S et al (2020) Development and validation of a model for individualized prediction of hospitalization risk in 4536 patients with COVID-19. PLoS One 15:e237419. https://doi.org/10.1371/journal.pone.0237419

18. Murillo-Zamora E, Trujillo X, Huerta M, Ríos-Silva M, MendozaCano O (2020) Male gender and kidney illness are associated with an increased risk of severe laboratory-confirmed coronavirus disease. BMC Infect Dis 20:674. https://doi.org/10.1186/ s12879-020-05408-6

19. Ortiz-Prado E, Simbana-Rivera K, Diaz AM et al (2020) Epidemiological, socio-demographic and clinical features of the early phase of the COVID-19 epidemic in Ecuador. MedRxiv 2020.05.08.20095943; https://doi.org/10.1101/2020.05.08.20095 943

20. Poblador-Plou B, Carmona-Pírez J, Ioakeim-Skoufa I et al (2020) Baseline chronic comorbidity and mortality in laboratory-confirmed COVID-19 cases: results from the PRECOVID study in Spain. Int J Environ Res Public Health 17:5171. https://doi.org/ 10.3390/ijerph17145171

21. Fink G, Orlova-Fink N, Schindler T, et al (2020) Inactivated trivalent influenza vaccine is associated with lower mortality among Covid-19 patients in Brazil n.d. https://www.medrxiv.org/ content/https://doi.org/10.1101/2020.06.29.20142505v1

22. Hui KPY, Cheung MC, Perera RAPM et al (2020) Tropism, replication competence, and innate immune responses of the coronavirus SARS-CoV-2 in human respiratory tract and conjunctiva: an analysis in ex-vivo and in-vitro cultures. Lancet Respir Med 8:687-695. https://doi.org/10.1016/S2213-2600(20)30193-4

23. Angelopoulou A, Alexandris N, Konstantinou E et al (2020) A toll like receptor 7 agonist-is an ideal option for management of COVID-19. Environ Res 188:109858. https://doi.org/10.1016/j. envres.2020.109858

24. Marín-Hernández D, Schwartz RE, Nixon DF (2020) Epidemiological evidence for association between higher influenza vaccine uptake in the elderly and lower COVID-19 deaths in Italy. J Med Virol. 2020 Jun 4:https://doi.org/10.1002/jmv.26120. Epub ahead of print. PMID: 32497290; PMCID: PMC7300995.

Publisher's Note Springer Nature remains neutral with regard to jurisdictional claims in published maps and institutional affiliations. 